\title{
Sagittal Pelvic Radius in Low-Grade Isthmic Lumbar Spondylolisthesis of Chinese Population
}

\author{
Yang Zhao, M.D., ${ }^{1}$ Cai-Liang Shen, M.D., Ph.D., ${ }^{1}$ Ren-Jie Zhang, M.D., ${ }^{1}$ Da-Wei Cheng, M.D., ${ }^{1}$ Fu-Long Dong, M.D., ${ }^{1}$ Jun Wang $^{2}$ \\ Departments of Spinal Surgery, ${ }^{1}$ Radiology, ${ }^{2}$ The First Affiliated Hospital of Anhui Medical University, Hefei, Anhui, China
}

\begin{abstract}
Objective : To investigate the variation of pelvic radius and related parameters in low-grade isthmic lumbar spondylolisthesis.
Methods : Seventy-four patients with isthmic lumbar spondylolisthesis and 47 controls were included in this study. There were 17 males and 57 females between 30 and 66 years of age, including 30 with grade I slippages and 44 grade II slippages; diseased levels included 34 cases on L4 and 40 cases on L5. Thoracic kyphosis (TK), the pelvic radius (PR), the pelvic angle (PA), pelvic morphology (PR-S1), and total lumbopelvic lordosis (PR-T12) were assessed from radiographs.

Results : Statistically significant differences were found for the PA, PR-T12, and PR-S1 $\left(24.5 \pm 6.6^{\circ}, 83.7 \pm 9.8^{\circ}\right.$, and $25.4 \pm 11.2^{\circ}$, respectively) of the patients with spondylolisthesis and the healthy volunteers $\left(13.7 \pm 7.8^{\circ}, 92.9 \pm 9.2^{\circ}\right.$, and $40.7 \pm 8.9^{\circ}$, respectively). The TK/PR-T12 ratios were between 0.15 and 0.75 . However, there were no differences in all the parameters between the L4 and L5 spondylolysis subgroups ( $p>0.05$ ). The TK and PR-S1 of grade II were less than grade I, but the PA was greater. The PR-T12 of female patients were less than male patients, but the PA was greater $(p<0.05)$.

Conclusion : Pelvic morphology differed in patients with low-grade isthmic lumbar spondylolisthesis compared to controls. Gender and the grade of slippage impacted the sagittal configuration of the pelvis, but the segment of the vertebral slip did not. Overall, the spine of those with spondylolisthesis remains able to maintain sagittal balance despite abnormal pelvic morphology.
\end{abstract}

Key Words : Isthmic lumbar spondylolisthesis $\cdot$ Pelvic radius $\cdot$ Sagittal balance.

\section{INTRODUCTION}

To stand upright, the human body must maintain a balance between spinal curvature and pelvic morphology ${ }^{1)}$. As the spine and the pelvis are connected in a biomechanical system ${ }^{4,20)}$, the morphology of pelvic curve will change to compensate for changes in spinal curvature.

When lumbar spondylolisthesis occurs, the sagittal shape of the pelvis will be inevitably affected. Recently, a few studies have reported the pelvic parameters in lumbar spondylolisthesis ${ }^{11,15,16,19)}$. However, the pelvic parameters only record local changes in pelvic morphology and do not take into account how the spine compensates for pelvic changes. Jackson et al. ${ }^{5,6)}$ notes that the pelvis and spine should be considered a whole unit when considering biomechanics, and for this, he proposed the pelvic radius (PR) technique, which reportedly has similar reliability to pelvic parameters ${ }^{7}$. Sergides notes that the $\mathrm{PR}$ technique can help to prevent poor visualization of $\mathrm{C}$, which is typical when using sagittal vertical axis from C7 plumb line (SVA) measurements ${ }^{18)}$. To the best of our knowledge, there are few studies assessing sagittal alignment in spondylolisthesis using the PR technique ${ }^{6}$. The aim of this study was to investigate the sagittal pelvic morphology in lumbar isthmic spondylolisthesis using the PR technique, compared to controls.

\section{MATERIALS AND METHODS}

\section{Patient population}

We retrospectively reviewed 74 Chinese patients who had a confirmed diagnosis of lumbar isthmic spondylolisthesis on Xray and a three-dimensional CT from January 2011 to April 2014. The local Institutional Review Board approved the study. The average age of the subjects was $49.2 \pm 9.5$ years (range : 30-66 years). There were 17 males and 57 females, which included 30 grade I slippages and 44 grade II slippages according to the system of Meyerding. They all had low-grade spondylolisthesis; 34

- Received : June 9, 2015 • Revised : December 23, 2015 •Accepted : February 13, 2016

- Address for reprints : Cai-Liang Shen, M.D., Ph.D.

Department of Spinal Surgery, The First Affiliated Hospital of Anhui Medical University, 218 Jixi Road, Hefei, Anhui 230022, China

Tel : +86-0551-62923244, Fax : +86-0551-62923244, E-mail : shencailiang1616@163.com

- This is an Open Access article distributed under the terms of the Creative Commons Attribution Non-Commercial License (http://creativecommons.org/licenses/by-nc/3.0) which permits unrestricted non-commercial use, distribution, and reproduction in any medium, provided the original work is properly cited. 
subjects had spondylolisthesis at L4 and 40 subjects at L5. The exclusion criteria were as follows : 1) previous spinal fracture or surgery, 2) spinal infection or tumor, 3) hip disease, and 4) lower limb deformities.

\section{Control group}

Healthy volunteers consisted of 23 females and 24 males with an average age of 23.3 years (range : $21-27$ years). They had no previous history of spinal, pelvic, or hip disorders. All subjects were college students. They were informed of the risks of participating in this study and signed an informed consent form.

\section{Radiographic parameters}

For each subject, the lateral view of spinal radiograph, including the base of the skull and the upper section of the femur, was obtained. Subjects were asked to stand upright with their knees extended, with their elbows bent to accommodate shoulder flexion to $30^{\circ 13)}$. The parameters using the PR technique included (Fig. 1) : 1) Pelvic radius (PR), the distance from the hip axis to the posterior-superior corner of the S1 endplate; the hip axis was located in the middle between the two femoral bead midpoints. 2) The pelvic angle (PA), the angle between the PR line and

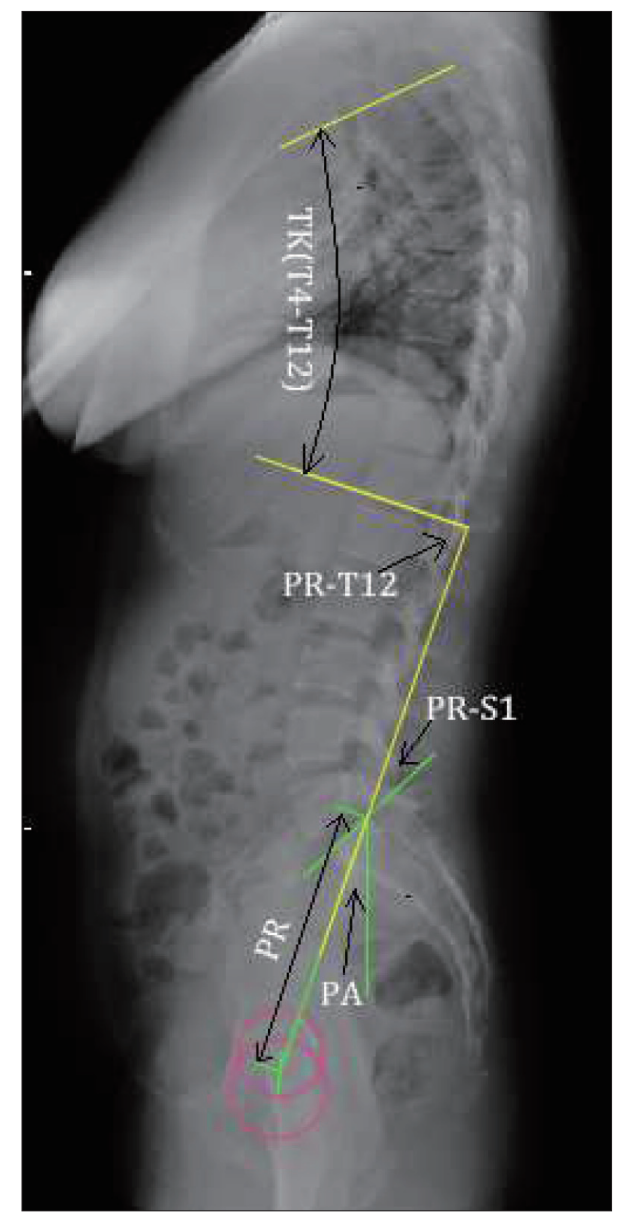

Fig. 1. Illustration showing the parameters included in this study : thoracic kyphosis (TK or T4-12), pelvic radius (PR), pelvic angle (PA), pelvic morphology (PR-S1) and total lumbopelvic Lordosis (PR-T12). the plumb line. 3) Pelvic morphology (PR-S1), the angle between the PR line and the S1 endplate line. 4) Total lumbopelvic lordosis (PR-T12) and thoracic kyphosis (TK), both T12-PR and T412 , measured using the Cobb technique.

\section{Statistical analysis}

Statistical analysis was performed using SPSS software (SPSS for Windows version 13.0, SPSS Inc., Chicago, IL, USA). We compared the demographic factors and spinal parameters of the patients and the healthy volunteers using an independent-samples t-test, with subgroups for gender, L4 and L5 spondylolisthesis, and $\mathrm{I}^{\circ}$ and $\mathrm{II}^{\circ}$ spondylolisthesis. The statistical significance level was set at $p<0.05$.

\section{RESULTS}

Compared to the control group, the TK $\left(37.2 \pm 9.1^{\circ}\right)$ and the PA $\left(24.5 \pm 6.6^{\circ}\right)$ of the total patients were significantly greater $(p<0.05)$, and the PR-S1 $\left(25.4 \pm 11.2^{\circ}\right)$ and PR-T12 $\left(83.7 \pm 9.8^{\circ}\right)$ were significantly lower $(p<0.05)$, while there was no significant difference in the PR (Table 1). However, the TK/PR-T12 ratio was consistently between 0.15 and 0.75 in the patient group.

The patient group was divided into those with grade I and grade II spondylolisthesis. The PA was significantly greater for grade I patients $\left(21.7 \pm 5.0^{\circ}\right)$ and grade II patients $\left(25.9 \pm 6.9^{\circ}\right)$ compared with the control group, respectively $\left(13.7 \pm 7.8^{\circ}\right)(p<0.05)$, and the PR-S1 and PR-T12 were significantly lower. The TK was significantly greater for grade I patients $\left(41.1 \pm 7.9^{\circ}\right)$ than that of the control group $\left(31.3 \pm 9.3^{\circ}\right)(p=0.001)$ (Table 2).

The patient group was divided into L4 and L5 spondylolisthesis subgroups. We compared the TK (T4-12), PR, PA, PR-S1, PRT12, and the TK/PR-T12 ratio between L4 and L5 subgroups, and found no statistical difference between the two groups ( $p>0.05)$, as shown in Table 3.

The TK and PR-S1 of grade II were less than grade I, but the PA was greater (Table 4$)$. The PR-T12 of female patients were less than male patients, but the PA was greater $(p<0.05)$ (Table 5).

\section{DISCUSSION}

The physiological curvature of the spine includes cervical lor-

Table 1. Comparison of the parameters of the patients and the control group

\begin{tabular}{lccc}
\hline \multicolumn{1}{c}{ Parameter } & $\begin{array}{c}\text { Control } \\
(\mathrm{n}=48)\end{array}$ & $\begin{array}{c}\text { Isthmic lumbar } \\
\text { spondylolisthesis }(\mathrm{n}=74)\end{array}$ & $p$ \\
\hline $\mathrm{PR}(\mathrm{mm})$ & $133.0 \pm 9.6$ & $131.9 \pm 8.9$ & 0.574 \\
$\mathrm{PA}\left(^{\circ}\right)$ & $13.7 \pm 7.8$ & $24.5 \pm 6.6$ & 0.000 \\
$\mathrm{PR}-\mathrm{S} 1\left(^{\circ}\right)$ & $40.7 \pm 8.9$ & $25.4 \pm 11.2$ & 0.000 \\
$\mathrm{PR}-\mathrm{T} 12\left(^{\circ}\right)$ & $92.9 \pm 9.2$ & $83.7 \pm 9.8$ & 0.000 \\
$\mathrm{TK}\left({ }^{\circ}\right)$ & $31.3 \pm 9.3$ & $37.2 \pm 9.1$ & 0.004 \\
T4-T12/PR-T12 & $0.34 \pm 0.11$ & $0.45 \pm 0.10$ & 0.000 \\
\hline
\end{tabular}

PR : pelvic radius, PA : pelvic angle, PR-S1 : pelvic morphology, PR-T12 : total lumbopelvic lordosis, TK and T4-12 : thoracic kyphosis 
Table 2. Comparison of the parameters of the control, $I^{\circ}$, and $\|^{\circ}$ subgroups

\begin{tabular}{lcrcrc}
\hline \multicolumn{1}{c}{ Parameter } & Control $(\mathrm{n}=47)$ & $\mathrm{I}^{\circ}(\mathrm{n}=30)$ & \multicolumn{1}{c}{$p$} & $\mathrm{II}(\mathrm{n}=44)$ & \multicolumn{1}{c}{$p$} \\
\hline $\mathrm{TK}(\mathrm{T} 4-12)\left(^{\circ}\right)$ & $31.3 \pm 9.3$ & $41.1 \pm 7.9$ & 0.001 & $35.2 \pm 9.1$ & 0.082 \\
$\mathrm{PR}(\mathrm{mm})$ & $133.0 \pm 9.6$ & $130.2 \pm 8.9$ & 0.333 & $132.7 \pm 8.9$ & 0.908 \\
$\mathrm{PA}\left({ }^{\circ}\right)$ & $13.7 \pm 7.8$ & $21.7 \pm 5.0$ & 0.001 & $25.9 \pm 6.9$ & 0.000 \\
$\mathrm{PR}-\mathrm{S} 1\left(^{\circ}\right)$ & $40.7 \pm 8.9$ & $30.3 \pm 9.9$ & 0.000 & $22.9 \pm 11.2$ & 0.000 \\
$\mathrm{PR}-\mathrm{T} 12\left(^{\circ}\right)$ & $92.9 \pm 9.2$ & $86.7 \pm 8.9$ & 0.030 & $82.2 \pm 10.1$ & 0.000 \\
\hline
\end{tabular}

PR : pelvic radius, PA : pelvic angle, PR-S1 : pelvic morphology, PR-T12 : total lumbopelvic lordosis, TK and T4-12 : thoracic kyphosis

Table 3. Comparison of the parameters of the $L 4$ and $L 5$ subgroups

\begin{tabular}{lccc}
\hline \multicolumn{1}{c}{ Parameter } & L4 $(\mathrm{n}=34)$ & $\mathrm{L} 5(\mathrm{n}=40)$ & $p$ \\
\hline $\mathrm{TK}(\mathrm{T} 4-12)\left(^{\circ}\right)$ & $38.6 \pm 9.6$ & $36.1 \pm 8.7$ & 0.388 \\
$\mathrm{PR}(\mathrm{mm})$ & $131.8 \pm 8.1$ & $131.9 \pm 9.5$ & 0.969 \\
$\mathrm{PA}\left({ }^{\circ}\right)$ & $26.1 \pm 6.8$ & $23.3 \pm 6.3$ & 0.175 \\
$\mathrm{PR}-\mathrm{S} 1\left(^{\circ}\right)$ & $27.5 \pm 12.1$ & $23.8 \pm 10.5$ & 0.298 \\
$\mathrm{PR}-\mathrm{T} 12\left(^{\circ}\right)$ & $81.6 \pm 11.4$ & $85.3 \pm 8.4$ & 0.233 \\
$\mathrm{~T} 4-12 / \mathrm{PR}-\mathrm{T} 12$ & $0.47 \pm 0.09$ & $0.43 \pm 0.10$ & 0.146 \\
\hline
\end{tabular}

PR : pelvic radius, PA : pelvic angle, PR-S1 : pelvic morphology, PR-T12 : total lumbopelvic lordosis, TK and T4-12 : thoracic kyphosis

Table 4. Comparison of the parameters of the $\mathrm{I}^{\circ}$ and $\mathrm{I}^{\circ}$ subgroups

\begin{tabular}{lccc}
\hline \multicolumn{1}{c}{ Parameter } & $\mathrm{I}^{\circ}(\mathrm{n}=30)$ & $\mathrm{II}{ }^{\circ}(\mathrm{n}=44)$ & $p$ \\
\hline $\mathrm{TK}(\mathrm{T} 4-12)\left(^{\circ}\right)$ & $41.1 \pm 7.9$ & $35.2 \pm 9.1$ & 0.044 \\
$\mathrm{PR}(\mathrm{mm})$ & $130.2 \pm 8.9$ & $132.7 \pm 8.9$ & 0.385 \\
$\mathrm{PA}\left({ }^{\circ}\right)$ & $21.7 \pm 5.0$ & $25.9 \pm 6.9$ & 0.049 \\
$\mathrm{PR}-\mathrm{S} 1\left(^{\circ}\right)$ & $30.3 \pm 9.9$ & $22.9 \pm 11.2$ & 0.040 \\
$\mathrm{PR}-\mathrm{T} 12\left(^{\circ}\right)$ & $86.7 \pm 8.9$ & $82.2 \pm 10.1$ & 0.160 \\
$\mathrm{~T} 4-12 / \mathrm{PR}-\mathrm{T} 12$ & $0.48 \pm 0.08$ & $0.43 \pm 0.11$ & 0.190 \\
\hline
\end{tabular}

PR : pelvic radius, PA : pelvic angle, PR-S1 : pelvic morphology, PR-T12 : total lumbopelvic lordosis, TK and T4-12 : thoracic kyphosis

Table 5. Comparison of the parameters of female and male subgroups

\begin{tabular}{lcrc}
\hline \multicolumn{1}{c}{ Parameter } & $\mathrm{F}(\mathrm{n}=17)$ & $\mathrm{M}(\mathrm{n}=57)$ & \multicolumn{1}{c}{$\mathrm{p}$} \\
\hline $\mathrm{TK}(\mathrm{T} 4-12)\left(^{\circ}\right)$ & $36.3 \pm 9.1$ & $40.6 \pm 8.4$ & 0.211 \\
$\mathrm{PR}(\mathrm{mm})$ & $131.6 \pm 8.8$ & $132.9 \pm 9.7$ & 0.711 \\
$\mathrm{PA}\left({ }^{\circ}\right)$ & $26.0 \pm 6.4$ & $19.1 \pm 3.9$ & 0.004 \\
$\mathrm{PR}-\mathrm{S} 1\left(^{\circ}\right)$ & $23.7 \pm 11.6$ & $31.4 \pm 7.7$ & 0.070 \\
PR-T12 $\left(^{\circ}\right)$ & $81.4 \pm 9.2$ & $92.0 \pm 7.7$ & 0.003 \\
T4-12/PR-T12 & $0.45 \pm 0.10$ & $0.44 \pm 0.09$ & 0.866 \\
\hline
\end{tabular}

PR : pelvic radius, PA : pelvic angle, PR-S1 : pelvic morphology, PR-T12 : total lumbopelvic lordosis, TK and T4-12 : thoracic kyphosis

dosis, thoracic kyphosis, lumbar lordosis, and sacral kyphosis. Normal physiological curvature is an important factor to ensure that people can walk upright and maintain balance, as the spine and the pelvis area work together as a unit to enable normal ambulation. However, spondylolisthesis inevitably leads to changes in the sagittal pelvic morphology.

Studying sagittal balance helps us to understand the changes in the lumbopelvic biomechanics in lumbar spondylolisthesis and provide theories to guide clinical treatment.

Most previous studies have focused on pelvic parameter ${ }^{11,16)}$. Since the spine and pelvis are a whole, when the sagittal alignment of the lumbar spine changes, the upper spine will make compensatory changes to regain balance". Therefore, the standalone use of pelvic parameters to evaluate the sagittal balance of lumbar spondylolisthesis may overlook the compensatory effect of the thoracic spine. Jackson et al. ${ }^{6}$ described a PR technique to evaluate sagittal spinopelvic balance, which includes PR, PA, PRT12, and PR-S1. They have reported that the reliability of PR-T12, PI, and PR-S1 is similar ${ }^{7}$. In addition to PR-S1, it is not necessary to identify the whole S1 endplate when use the PR technique to measure. Using PI and SVA methods, clear visualization of the S1 endplate and the C7 vertebra is essential. But according to reports, the $\mathrm{C} 7$ vertebrae can be clearly identified on the whole spine lateral radiographs only in about half of the cases ${ }^{18)}$. Our study also found that the $\mathrm{C} 7$ vertebrae could not be fully visualized in most patients. Using the PR techniques, we can obtain more accurate data than the PI and SVA methods. Jackson et al. ${ }^{7}$ indicated that the PR technique was more reliable than plumbline measurement.

Meanwhile, Jackson et al..$^{5,7)}$ proposed the concept of "congruent" spinopelvic alignment. "Congruent" alignment should meet the following three conditions : 1) PR-T12 is between $70^{\circ}$ and $\left.100^{\circ}, 2\right)$ the PA is between $3^{\circ}$ and $33^{\circ}$, and 3) the T4-12/PR-T12 ratio is between 0.15 and 0.75 .

Geometrically, PR-T12 is the sum of PR-S1 and T12-S1. Its value is in a relatively narrow range in healthy individuals, about $90^{\circ} \pm 10^{\circ 18}$. Thus, the total lumbopelvic lordosis decreases when the PR-S1 increases, and vice versa.

We found that the PA of spondylolisthesis was significantly increased, while the PR-S1 and PR-T12 were significantly reduced, and, as the degree of spondylolisthesis increased, the PA increased, but the PR-S1 decreased. Therefore, with an increasing degree of spondylolisthesis, the pelvis rotates around the hip axis, leading to verticalization of the sacrum ${ }^{17}$. Meanwhile, we found that the PA of females is greater than that of males, indicating that the female pelvis tilts backward seriously. Thus, the shear force of the female lumbosacral part is greater ${ }^{16)}$ and the probability of the occurrence of spondylolisthesis is also greater.

Park et al. ${ }^{15)}$ observed that lumbar spondylolisthesis can affect the sagittal balance of the lumbar spine. Sergides et al. ${ }^{18)}$ pointed out that although PR-T12 can quickly evaluate sagittal lumbar balance, the compensatory effect of the upper spine cannot be ignored.

We found that the PA, PR-S1, and PR-T12 of low-grade spondylolisthesis differed from the normal ranges significantly, dem- 
onstrating that the local morphology of the lumbar spine and pelvis have changed. However, according to the "congruent" spinopelvic alignment criteria ${ }^{7)}$, for low-grade spondylolisthesis patients, the sagittal trunk is still in a state of balance. This demonstrates that when the local morphology of the lumbar spine and pelvis changes, the remainder of the spine will compensate in terms of position to maintain overall balance, such as intervertebral hyperextension and pelvic retroversion ${ }^{10)}$. Therefore, the PR technique enables effective evaluation of spinal sagittal balance and minimizes the measurement error caused by unclear views of $\mathrm{C7}$.

Because the L4/L5 vertebrae are the apex of lumbar lordosis ${ }^{2)}$, the lower lumbar spine is a predominant region for biomechanical transmission, and thus lumbar spondylolisthesis often occurs in this region. Oh et al. ${ }^{14)}$ observed that spondylolisthesis of different segments of the lower lumbar spine incurs similar changes in pelvic morphology. We observed similar results in our study : there was no significant difference in the PR and related parameters between L4 and L5 spondylolisthesis. For severe spondylolisthesis (slip percentage $>50 \%$ ), the difference in the pelvic morphological changes of different segments of spondylolisthesis requires further investigation.

Mac-Thiong et al. ${ }^{12)}$ indicated that sagittal imbalance is more likely to cause pain and dysfunction. Posterior pedicle screw fixation is the major surgical treatment for spondylolisthesis. By restoring sagittal balance, it can reduce the incidence of flat back syndrome and pathology in the adjacent segments ${ }^{8}$, thus achieving a better prognosis ${ }^{3,20)}$. Satisfactory clinical outcomes are dependent on preoperative measurements, which can help us to adjust the angle of pedicle screws during operations and restore sagittal spinopelvic balance.

Our study had several limitations, such as a small number of cases; in addition, we did not include severe spondylolisthesis and multiple-level spondylolisthesis, which may affect the results.

\section{CONCLUSION}

Pelvic morphology is abnormal in spondylolisthesis, but spinopelvic balance is not affected. The gender and grade of slippage had an effect on the sagittal configuration of the pelvis, but there are no differences in terms of the impacts different segments of vertebral slip had on pelvic morphology.

\section{References}

1. Barrey C, Roussouly P, Perrin G, Le Huec JC : Sagittal balance disorders in severe degenerative spine. Can we identify the compensatory mechanisms? Eur Spine J 20 Suppl 5 : 626-633, 2011

2. Berthonnaud E, Dimnet J, Roussouly P, Labelle H : Analysis of the sagittal balance of the spine and pelvis using shape and orientation parame- ters. J Spinal Disord Tech 18 : 40-47, 2005

3. Bourghli A, Aunoble S, Reebye O, Le Huec JC : Correlation of clinical outcome and spinopelvic sagittal alignment after surgical treatment of low-grade isthmic spondylolisthesis. Eur Spine J 20 Suppl 5 : 663-668, 2011

4. Chanplakorn P, Wongsak S, Woratanarat P, Wajanavisit W, Laohacharoensombat $\mathrm{W}$ : Lumbopelvic alignment on standing lateral radiograph of adult volunteers and the classification in the sagittal alignment of lumbar spine. Eur Spine J 20 : 706-712, 2011

5. Jackson RP, Hales C : Congruent spinopelvic alignment on standing lateral radiographs of adult volunteers. Spine (Phila Pa 1976) 25 : 28082815, 2000

6. Jackson RP, Peterson MD, McManus AC, Hales C : Compensatory spinopelvic balance over the hip axis and better reliability in measuring lordosis to the pelvic radius on standing lateral radiographs of adult volunteers and patients. Spine (Phila Pa 1976) 23 : 1750-1767, 1998

7. Jackson RP, Phipps T, Hales C, Surber J : Pelvic lordosis and alignment in spondylolisthesis. Spine (Phila Pa 1976) 28 : 151-160, 2003

8. Kim CH, Chung CK, Park SB, Yang SH, Kim JH : A change in lumbar sagittal alignment after single-level anterior lumbar interbody fusion for lumbar degenerative spondylolisthesis with normal sagittal balance. J Spinal Disord Tech, 2014 [Epub ahead of print]

9. Kim YB, Kim YJ, Ahn YJ, Kang GB, Yang JH, Lim H, et al. : A comparative analysis of sagittal spinopelvic alignment between young and old men without localized disc degeneration. Eur Spine J 23 : 1400-1406, 2014

10. Labelle H, Mac-Thiong JM, Roussouly P : Spino-pelvic sagittal balance of spondylolisthesis : a review and classification. Eur Spine J 20 Suppl 5 : 641-646, 2011

11. Lim JK, Kim SM : Difference of sagittal spinopelvic alignments between degenerative spondylolisthesis and isthmic spondylolisthesis. J Korean Neurosurg Soc 53 : 96-101, 2013

12. Mac-Thiong JM, Transfeldt EE, Mehbod AA, Perra JH, Denis F, Garvey TA, et al. : Can c7 plumbline and gravity line predict health related quality of life in adult scoliosis? Spine (Phila Pa 1976) 34 : E519-E527, 2009

13. Marks M, Stanford C, Newton P : Which lateral radiographic positioning technique provides the most reliable and functional representation of a patient's sagittal balance? Spine (Phila Pa 1976) 34: 949-954, 2009

14. Oh SK, Chung SS, Lee CS : Correlation of pelvic parameters with isthmic spondylolisthesis. Asian Spine J 3 : 21-26, 2009

15. Park SJ, Lee CS, Chung SS, Kang KC, Shin SK : Postoperative changes in pelvic parameters and sagittal balance in adult isthmic spondylolisthesis. Neurosurgery 68 (2 Suppl Operative) : 355-363; discussion 362-363, 2011

16. Rajnics P, Templier A, Skalli W, Lavaste F, Illés T : The association of sagittal spinal and pelvic parameters in asymptomatic persons and patients with isthmic spondylolisthesis. J Spinal Disord Tech 15 : 24-30, 2002

17. Roussouly P, Pinheiro-Franco JL : Sagittal parameters of the spine : biomechanical approach. Eur Spine J 20 Suppl 5 : 578-585, 2011

18. Sergides IG, McCombe PF, White G, Mokhtar S, Sears WR : Lumbo-pelvic lordosis and the pelvic radius technique in the assessment of spinal sagittal balance : strengths and caveats. Eur Spine J 20 Suppl 5 : 591-601, 2011

19. Vialle R, Ilharreborde B, Dauzac C, Lenoir T, Rillardon L, Guigui P : Is there a sagittal imbalance of the spine in isthmic spondylolisthesis? A correlation study. Eur Spine J 16 : 1641-1649, 2007

20. Wang TP, Zheng ZM, Liu H, Zhang KB, Wang H : [Correlation of adult spinal sagittal imbalance and life quality]. Zhonghua Yi Xue Za Zhi 92 : 1481-1485, 2012 